\title{
Efficient Frequency Invariant Beamforming using Virtual Arrays
}

\author{
Piya Pal, and P. P. Vaidyanathan \\ Dept. of Electrical Engineering, MC 136-93 \\ California Institute of Technology, Pasadena, CA 91125, USA \\ E-mail: piyapal@caltech.edu,ppvnath@systems.caltech.edu
}

\begin{abstract}
In wideband array processing, frequency invariant beamforming provides a popular means to make the beampattern allpass with respect to frequency. Traditionally, such beampatterns are realized as a two dimensional filter, using tapped delay-line (TDL) filters following each spatial sensor. However it has been recently shown that with the help of a rectangular antenna array, it is possible to generate fixed frequency invariant beampatterns without using filters. In this paper, this concept is generalized to the case of two dimensional arrays with elements on a (possibly nonseparable) lattice. Since performance of the frequency invariant beamformer depends on the number of sensors which could be large for a $2 D$ array of size $M \times N$, a novel approach to beamforming based on the difference coarray of a physical array is also proposed, which avoids use of additional physical sensors. The realization of the frequency invariant beams using second order statistics of the impinging signal with only $M+N$ physical sensors, instead of the two dimensional array of size $M \times N$, is demonstrated. The usefulness of the proposed method is verified through computer simulation.
\end{abstract}

Index Terms - Frequency Invariant Beamforming, Two Dimensional Array, Lattice, Difference co-array.

\section{INTRODUCTION}

In array processing for wideband signals, the temporal frequency and the spatial angle of the impinging signals are usually coupled together in the phase, which makes the resulting beampattern a function of both frequency and spatial angle. Frequency invariant beamforming is often used to make the beampattern approximately independent with respect to the temporal frequency and it has been studied extensively in [1], [7], [6], [10], [12]-[14]. Several techniques exist in the literature discussing generation of these frequency invariant beams [1], [7], [10], [13]. However, all of them either use filters or frequency dependent weights following each sensor.

In [2], however, it has been shown that frequency invariant beamforming can be achieved using just one weight per sensor instead of tapped delay-lines, by extending the dimension of the array from linear to rectangular. In this paper, we generalize this concept to the case of more generic two dimensional arrays with elements on a possibly non separable lattice (as opposed to the rectangular array which is an example of a separable lattice) and demonstrate how to perform frequency invariant beamforming by using the extra spatial dimension provided by these lattices, to eliminate temporal processing.

The performance of the frequency invariant beamformers however depends on the size of the array and for a two dimensional array, it could mean the use of a large number of sensors. In this paper, a novel solution to this is also proposed

${ }^{1}$ Work supported in parts by the ONR grant N00014-08-1-0709, and the California Institute of Technology. by using the so called difference co-array [3] of a physical array. It is demonstrated that using two suitably oriented non collinear (e.g., orthogonal) uniform linear arrays (ULAs), a desired two dimensional array can be realized virtually, by taking the cross difference co-array of these two arrays. To implement this from the physical model, we adopt a non linear approach to beamforming, which, instead of filtering the amplitudes, performs spatial filtering of the power of the impinging signals and is capable of exploiting the virtual degrees of freedom. We proposed the narrowband version of this beamforming in [8] using a nested array structure which was linear. A similar approach to beamforming using time averaged arrays was proposed in [4], [5]. However, in this paper, instead of realizing a long linear array, we need the additional degrees of freedom in the form of a two dimensional array to eliminate TDL filtering in wideband frequency invariant beamforming.

The rest of the paper is organized as follows. In Section II, we introduce the theory of frequency invariant beamforming using two dimensional arrays with elements on a lattice. In Section III, we prove a lemma regarding generation of coarrays with elements on a lattice. We then propose the design of frequency invariant beams using the difference co-array of suitable combination of ULAs, to gain the same $O\left(N^{2}\right)$ degrees of freedom of a lattice using only $O(N)$ physical sensors. We also provide numerical examples to support the proposed methods. Finally, Section IV concludes the paper.

\section{THEORY OF LATTICE BASED FREQUENCY INVARIANT BEAMFORMING WITHOUT FILTERS}

For a two dimensional array with sensors on a lattice, the position vector of the $(m, n)$ th sensor is given by

$$
\left(\begin{array}{l}
r_{x} \\
r_{y}
\end{array}\right)=\left[\begin{array}{ll}
\mathbf{v}_{\mathbf{1}} & \mathbf{v}_{\mathbf{2}}
\end{array}\right]\left[\begin{array}{c}
m \\
n
\end{array}\right]
$$

where $\mathbf{v}_{\mathbf{1}}$ and $\mathbf{v}_{\mathbf{2}}$ are independent vectors of size $2 \times 1$ and $m$ and $n$ are integers. Consider such an array of size $M \times N$ and let a wideband signal $s^{(c)}(t)$ impinge on it from an elevation angle $\phi$ and azimuthal angle $\theta$. The signal received at the $(m, n)$ th antenna, after demodulation of the carrier frequency $f_{c}$, is given by

$$
x_{m, n}^{(c)}(t)=s^{(c)}\left(t-\tau_{m, n}\right) e^{-j 2 \pi f_{c} \tau_{m, n}},
$$

where

$$
\tau_{m, n}=\frac{1}{c}\left[\begin{array}{ll}
\sin \phi \cos \theta & \sin \phi \sin \theta
\end{array}\right] \mathbf{V}\left[\begin{array}{c}
m \\
n
\end{array}\right] .
$$

Here $c$ is the propagation velocity. After sampling at period of $T_{s}$ (where $T_{s}$ is less than the Nyquist interval for $s^{(c)}(t)$ ), 
the discrete-time Fourier Transform (DTFT) of the $(m, n)$ th sensor output, is given by

$$
X_{m, n}\left(e^{j \omega}, \theta\right)=e^{-j \frac{\hat{\omega}}{T_{s}} \tau_{m, n}} S\left(e^{j \omega}\right)
$$

where $S\left(e^{j \omega}\right)$ is the DTFT of samples of $s^{(c)}(t)$, and $\widehat{\omega}=$ $\omega+2 \pi f_{c} T_{s}$. Signal received at the $(m, n)$ th sensor is then multiplied by the weight $C_{m, n}$ and then all these signals from $M N$ sensors are summed up to get the output signal

$$
Y\left(e^{j \omega}, \theta\right)=H\left(e^{j \omega}, \theta\right) S\left(e^{j \omega}\right)
$$

where

$$
H\left(e^{j \omega}, \theta\right)=\sum_{m=0}^{M-1} \sum_{n=0}^{N-1} C_{m, n} e^{-j \frac{\hat{\omega}}{T_{s}} \tau_{m, n}}
$$

is the beampattern which is a function of both temporal frequency $\omega$ and spatial direction $\theta$. Our aim is to make this beampattern frequency invariant, i.e., allpass with respect to $\omega$ :

$$
H\left(e^{j \omega}, \theta\right)=G(\theta)
$$

where $G(\theta)$ is a desired narrowband beampattern that we wish to realize. The usual approach to achieve this with a ULA will be to make each of the weights $C_{m, n}$ a function of frequency itself, i.e., apply a TDL filter at the output of each sensor. However, since we are considering two dimensional arrays, the extra spatial dimension can be used to avoid the temporal dimension, thereby making each $C_{m, n}$ a constant weight instead of TDL filter.

Assuming $\mathbf{V}=\left[\begin{array}{cc}v_{11} & v_{12} \\ v_{21} & v_{22}\end{array}\right]$ and $\phi=90^{\circ}$ (as in [2] ), we define two new variables as

$$
\begin{aligned}
& \omega_{1}=\frac{\hat{\omega}}{c T_{s}}\left(v_{11} \cos \theta+v_{21} \sin \theta\right) \\
& \omega_{2}=\frac{\hat{\omega}}{c T_{s}}\left(v_{12} \cos \theta+v_{22} \sin \theta\right)
\end{aligned}
$$

Then, we can rewrite (5) as

$$
G(\theta)=G_{1}\left(\omega_{1}, \omega_{2}\right)=\sum_{m=0}^{M-1} \sum_{n=0}^{N-1} C_{m, n} e^{-j m \omega_{1}} e^{-j n \omega_{2}}
$$

Thus $G(\theta)$ can be represented as the two dimensional Fourier transform of the weights $C_{m, n}$ in the variables $\omega_{1}$ and $\omega_{2}$ and hence, theoretically, we can realize $G(\theta)$ by computing the weights $C_{m, n}$ through inverse DFT as

$$
C_{m, n}=\frac{1}{M N} \sum_{k=0}^{M-1} \sum_{l=0}^{N-1} G_{1}\left(\omega_{1 k}, \omega_{2 l}\right) e^{j m \omega_{1 k}} e^{j n \omega_{2 l}},
$$

where $\omega_{1 k}=-\pi+\frac{2 \pi k}{M}, \omega_{2 l}=-\pi+\frac{2 \pi l}{N}$.

Hence, this technique provides a direct way to compute the weights $C_{m, n}$ and it guarantees to approximate the desired frequency invariant beampattern at the sample points due to the FFT relation.

\section{Passive Realization of the Lattice BASED} FREQUENCY INVARIANT BEAMFORMING USING CO-ARRAY

So far it has been demonstrated that in order to obtain a frequency invariant beampattern, either (a) a linear array followed by temporal processing, or, (b) a two dimensional array with no temporal processing, is needed. The need for temporal processing is avoided by the corresponding increase in spatial dimension of the array. The original beampattern in $(\omega, \theta)$ domain, is mapped to a transformed domain $\left(\omega_{1}, \omega_{2}\right)$ given by (7)-(8). In order to realize the frequency invariant beam, we need to represent (9) entirely in terms of $\omega_{1}$ and $\omega_{2}$ without the temporal frequency $\omega$. This is equivalent to eliminating $\hat{\omega}$ between (7) and (8) for which we need these equations to be independent. When the array is linear, the equations are no more independent and hence the spatial-only approach to frequency invariant beamforming fails. We shall demonstrate this by describing a specific example of linear array, viz., the nested array (proposed in [8]) later to show that though such arrays result in increased degrees of freedom, they cannot be used to realize frequency invariant beamforming because the freedoms are in the form of virtual sensors which form a linear array. In other words $\mathbf{V}$ has rank less than 2 . Hence, the increase in spatial dimension is necessary.

In this section, we propose a novel method to create the effect of the same two dimensional array without using the additional number of sensors. This will be achieved by a combination of the following two techniques:

- A new "non linear" approach to beamforming which filters the power spectrum of the impinging wideband signals.

- Suitable placement of physical sensors to create the effect of the desired two dimensional array by considering "cross difference co-array" of two suitable ULAs. Some special types of cross product arrays have been discussed earlier in [9].

We first define the difference co-array of a physical array and then demonstrate how it helps in realization of a virtual array.

Definition 1: (Difference Co-array) Let us consider an array of $N$ sensors, with $\overrightarrow{\mathbf{x}_{i}}$ denoting the position vector of the $i$ th sensor. Define the set

$$
D=\left\{\overrightarrow{\mathbf{x}_{i}}-\overrightarrow{\mathbf{x}_{j}}\right\}, \quad \forall i, j=1,2, \cdots, N
$$

In our definition of the set $D$, we allow repetition of its elements. We also define the set $D_{u}$ which consists of the distinct elements of the set $D$. Then, the difference co-array of the given array is defined as the array which has sensors located at positions given by the set $D_{u}$.

It can be verified [8] that the maximum degrees of freedom obtainable from a difference co-array for a $N$ element array with any geometry and dimension, is

$$
\mathrm{DOF}_{\max }=N(N-1)+1 .
$$

Details about the different kinds of co-arrays of a physical array and its various applications can be found in [3].

\section{A. Wideband Beamforming Based on Difference Co-array:}

We now demonstrate how to get the effect of the difference co-array in wideband beamforming by using a "non linear" approach to beamforming. The narrowband version of this 
beamforming was proposed in [8]. An idea similar to this was discussed in [4], [5] where beamforming based on timeaveraged arrays was suggested using specific array geometries. This kind of beamforming can increase the degrees of freedom of a $N$ element physical array to $O\left(N^{2}\right)$.

Consider $D$ wideband signals, occupying frequency band $\omega_{a}$ to $\omega_{b}$, impinging on an array of $N$ sensors. Then, the signal received at the $n$th sensor is given by

$$
x_{n}(t)=\sum_{i=1}^{D} s_{i}\left(t-\tau_{n}\left(\theta_{i}\right)\right)
$$

where $\tau_{n}\left(\theta_{i}\right)$ is the delay associated with the $n$th array element and the $i$ th source signal and depends on the angle of arrival of the signal and the array geometry. For simplicity, we only consider the azimuthal angle $\theta$ though the delay is also a function of the elevation angle $\phi$ which we set to $90^{\circ}$ as earlier. Defining $\mathbf{x}(t)=\left[\begin{array}{ll}x_{1}(t) & x_{2}(t) \cdots x_{N}(t)\end{array}\right]^{T}$, and taking the autocorrelation of $\mathbf{x}(t)$, we get

$$
\mathbf{R}_{\mathbf{x} \mathbf{x}}=E\left(\mathbf{x}(t) \mathbf{x}^{H}(t)\right)
$$

where the $(m, n)$ th element of $\mathbf{R}_{\mathbf{x x}}$ is given by

$$
\begin{aligned}
{\left[\mathbf{R}_{\mathbf{x x}}\right]_{m, n} } & =E\left(x_{m}(t) x_{n}^{*}(t)\right) \\
& =E\left[\sum_{i=1}^{D} s_{i}\left(t-\tau_{m}\left(\theta_{i}\right)\right) \sum_{j=1}^{D} s_{j}^{*}\left(t-\tau_{n}\left(\theta_{j}\right)\right)\right]
\end{aligned}
$$

Assuming the sources are uncorrelated (i.e., $E\left[s_{i}(t) s_{j}^{*}(t-\right.$ $\tau)]=0, \quad \forall \quad \tau, \quad i \neq j$ ) we can rewrite (14) as

$$
\left[\mathbf{R}_{\mathbf{x x}}\right]_{m, n}=\sum_{i=1}^{D} R_{s_{i}, s_{i}}\left(\tau_{n}\left(\theta_{i}\right)-\tau_{m}\left(\theta_{i}\right)\right)
$$

where $R_{s_{i}, s_{i}}(\tau)$ denotes the autocorrelation of the $i$ th signal. Now, we vectorize $\mathbf{R}_{\mathbf{x x}}$ to get the vector $\mathbf{y} \in \mathcal{C}^{N^{2} \times 1}$. Treating this as the equivalent signal vector (corresponding to the co-array), we wish to apply weights to this vector to perform spatial filtering. Denoting the weight vector as $\mathbf{w} \in \mathcal{C}^{N^{2} \times 1}$, we index its elements by the double index $(m, n), m=0, \cdots, N-1, n=0, \cdots, N-1$ for convenience. Then, assuming the sources are bandlimited to $\omega_{a} \leq \omega \leq \omega_{b}$, the final weighted output can be written as

$$
\begin{aligned}
z & =\mathbf{w}^{H} \mathbf{y} \\
& =\sum_{i=1}^{D} \sum_{m=0}^{N-1} \sum_{n=0}^{N-1} w_{m, n} R_{s_{i}, s_{i}}\left(\tau_{n}\left(\theta_{i}\right)-\tau_{m}\left(\theta_{i}\right)\right) \\
& =\sum_{i=1}^{D} \sum_{m=0}^{N-1} \sum_{n=0}^{N-1} w_{m, n} \frac{1}{2 \pi} \int_{\omega_{a}}^{\omega_{b}} P_{s_{i}, s_{i}}(\omega) e^{j \omega\left(\tau_{n}\left(\theta_{i}\right)-\tau_{m}\left(\theta_{i}\right)\right)} d \omega
\end{aligned}
$$

where $P_{s_{i}, s_{i}}(\omega)$ denotes the power spectral density of $s_{i}(t)$. We can further rewrite (18) as

$$
\begin{aligned}
z & =\frac{1}{2 \pi} \sum_{i=1}^{D} \int_{\omega_{a}}^{\omega_{b}}\left(\sum_{m=0}^{N-1} \sum_{n=0}^{N-1} w_{m, n} e^{j \omega\left(\tau_{n}\left(\theta_{i}\right)-\tau_{m}\left(\theta_{i}\right)\right)}\right) P_{s_{i}, s_{i}}(\omega) d \omega \\
& =\frac{1}{2 \pi} \sum_{i=1}^{D} \int_{\omega_{a}}^{\omega_{b}} B\left(\omega, \theta_{i}\right) P_{s_{i}, s_{i}}(\omega) d \omega
\end{aligned}
$$

where

$$
B\left(\omega, \theta_{i}\right) \triangleq \sum_{m=0}^{N-1} \sum_{n=0}^{N-1} w_{m, n} e^{j \omega\left(\tau_{n}\left(\theta_{i}\right)-\tau_{m}\left(\theta_{i}\right)\right)}
$$

is the beampattern (function of both temporal frequency $\omega$ and the spatial angle $\theta$ ). It is to be noted from (20) that the beampattern $B\left(\omega, \theta_{i}\right)$ filters the power spectrum $\left(P_{s_{i}, s_{i}}\right)$ of the signal instead of the amplitude. So it is essentially nonlinear in nature (linear with respect to the PSD). Also, though it appears that the beampattern has $N^{2}$ design freedoms in the form of the $N^{2}$ weights $w_{m, n}$, not all are independent. The number of free-to-choose weights are decided by the number of distinct phase differences $\tau_{n}\left(\theta_{i}\right)-\tau_{m}\left(\theta_{i}\right)$ which is completely determined by the number of distinct elements in the difference co-array, since

$$
\tau_{n}\left(\theta_{i}\right)-\tau_{m}\left(\theta_{i}\right)=\frac{1}{c} \mathbf{u}^{T}\left(\theta_{i}\right)\left(\mathbf{x}_{m}-\mathbf{x}_{n}\right) .
$$

Here $\mathbf{x}_{m}$ denotes the position vector of the $m$ th sensor and hence $\mathbf{x}_{m}-\mathbf{x}_{n}$ corresponds to the difference co-array element and $\mathbf{u}\left(\theta_{i}\right)=\left[\begin{array}{ll}\cos \theta_{i} & \sin \theta_{i}\end{array}\right]^{T}$ is the wave propagation vector. For example, if the array is a ULA, then the difference set has only $2 N-1$ distinct elements and so the effective number of weights in (21) free to be designed is only $2 N-1$ instead of $N^{2}$. In the two level nested array proposed in [8], the difference set has $\left(N^{2}-2\right) / 2+N$ degrees of freedom for even $N$ and $\left(N^{2}-1\right) / 2+N$ degrees of freedom for odd $N$ and the difference co-array is also in the form of a ULA. Hence, by suitable selection of the original physical array, $O\left(N^{2}\right)$ degrees of freedom in the same dimension can be obtained from $O(N)$ physical sensors.

Our goal here is to use these extra spatial freedoms to realize a frequency invariant beampattern, i.e., achieve

$$
B(\omega, \theta)=G(\theta), \quad \omega_{a} \leq \omega \leq \omega_{b}
$$

where $G(\theta)$ is a desired frequency invariant beampattern (e.g., a narrowband beampattern). In this case, we can rewrite (20) as

$$
\begin{aligned}
z & =\sum_{i=1}^{D} G\left(\theta_{i}\right) \frac{1}{2 \pi} \int_{\omega_{a}}^{\omega_{b}} P_{s_{i}, s_{i}}(\omega) d \omega \\
& =\sum_{i=1}^{D} G\left(\theta_{i}\right) \sigma_{i}^{2}
\end{aligned}
$$

where $\sigma_{i}^{2}$ denotes the power of the $i$ th source signal. Taking the additive noise at each antenna also into account, and assuming that it is spatially and temporally white, and uncorrelated with the signals, the final output becomes

$$
z=\sum_{i=1}^{D} G\left(\theta_{i}\right) \sigma_{i}^{2}+\sigma_{n}^{2} \mathbf{w}^{H} \tilde{\mathbf{1}}_{n},
$$

where $\sigma_{n}^{2}$ denotes the noise power, which has the same beamformer output as the corresponding narrowband beamformer proposed in [8].

To get the effect of frequency invariant beamforming as implemented using spatial only processing with a two dimensional array, we require the $N^{2}$ phase differences in (21) to correspond to those of a $N \times N$ two dimensional array. This is not possible using only one physical "linear" array (like the ULA or the nested array) because the difference co-array 
will always be linear and hence the weights $w_{m, n}$ need to be functions of frequency (TDL filters) for realizing the frequency invariant beam. To get the effect of a two dimensional array, the sensors need to be suitably placed, as described in the following subsection. It is to be noted that in order to eliminate the cross correlation between the impinging sources and obtain (15), sufficient number of snapshots is required.

\section{B. Realization of the lattice as the difference co-array}

In this section, we prove a lemma which will enable us to realize a two dimensional lattice virtually with only two suitably constructed one dimensional antenna arrays by using the cross difference co-array between them:

Lemma 1: Any 2-dimensional antenna array of size $M \times N$ with elements on a lattice, can be realized using the difference co-array of two uniform linear arrays with $M$ and $N$ elements respectively.

Proof: Let us consider a 2-D lattice in the $X Y$ plane described by the lattice generator matrix $\left(\begin{array}{lll}\mathbf{v}_{\mathbf{0}} & \mathbf{v}_{\mathbf{1}}\end{array}\right)$. Then the position vector of the $(m, n)$ th sensor is given by $\mathbf{v}_{0} m+$ $\mathbf{v}_{\mathbf{1}} n$. Now let us consider two ULAs with $M$ antennas and $N$ antennas respectively, the first ULA being in the same direction as the vector $-\mathbf{v}_{\mathbf{0}}$ and the second being in the same direction as $\mathbf{v}_{\mathbf{1}}$. In other words, the position vector of the $m$ th element of this ULA is $\tilde{\rho}_{\mathbf{1 , \mathbf { m }}}=-m \mathbf{v}_{\mathbf{0}}, \quad m=0,1 \cdots M-1$, and the position vector of the $n$th element of this second array is $\tilde{\rho}_{\mathbf{2}, \mathbf{n}}=n \mathbf{v}_{\mathbf{1}}, \quad n=0,1 \cdots N-1$. The cross difference coarray of these two arrays is defined as the array with position vectors

$$
\begin{gathered}
\overrightarrow{\mathbf{p}}_{m, n}=\tilde{\rho}_{\mathbf{2 , \mathbf { n }}}-\tilde{\rho}_{\mathbf{1}, \mathbf{m}} \\
m=0,1, \cdots, M-1, n=0,1, \cdots, N-1
\end{gathered}
$$

which can be readily seen as

$$
\overrightarrow{\mathbf{p}}_{m, n}=m \mathbf{v}_{\mathbf{0}}+n \mathbf{v}_{\mathbf{1}}
$$

corresponding to the $(m, n)$ th sensor of the lattice.

\section{Frequency Invariant Beamforming using the difference} array:

To physically realize the difference co-array corresponding to the lattice, we follow the covariance matrix based beamforming approach discussed earlier. However, instead of considering the self-difference co-array, we now consider the cross difference co-array of the two ULAs described in the preceding lemma. Let $\mathbf{x}_{\mathbf{1}}(t) \in \mathcal{C}^{M \times 1}$ and $\mathbf{x}_{\mathbf{2}}(t) \in \mathcal{C}^{N \times 1}$ denote the signal vectors received at the $M$-element and $N$ element ULAs oriented along $-\mathbf{v}_{\mathbf{0}}$ and $\mathbf{v}_{\mathbf{1}}$ respectively. So, the $m$ th element of $\mathbf{x}_{\mathbf{1}}(t)$ is given by

$$
\begin{aligned}
{\left[\mathbf{x}_{\mathbf{1}}\right]_{m}(t)=\sum_{i=1}^{D} s_{i}\left(t+m \mathbf{u}\left(\theta_{i}\right)^{T} \mathbf{v}_{\mathbf{0}}\right)+n_{m}(t), } & \\
& m=0,1, \cdots, M-1
\end{aligned}
$$

where $\mathbf{u}(\theta)$ denotes the propagation vector. Similarly, the $n$th element of $\mathbf{x}_{2}(t)$ is given by

$$
\begin{aligned}
{\left[\mathbf{x}_{\mathbf{2}}\right]_{n}(t)=\sum_{i=1}^{D} s_{i}\left(t-n \mathbf{u}\left(\theta_{i}\right)^{T} \mathbf{v}_{\mathbf{1}}\right)+} & n_{n}(t), \\
n & =0,1, \cdots, N-1
\end{aligned}
$$

The noise term $n_{m}(t)$ is assumed white Gaussian, which is spatially and temporally uncorrelated with same power for all the antennas in both arrays.

Next, we take the cross correlation of the signals received at the two ULAs, i.e.,

$$
\mathbf{R}_{\mathbf{x}_{\mathbf{1}}, \mathbf{x}_{\mathbf{2}}}=E\left[\mathbf{x}_{\mathbf{1}}(t) \mathbf{x}_{\mathbf{2}}{ }^{H}(t)\right]
$$

The $(m, n)$ th entry of the cross correlation matrix is given by

$$
\begin{aligned}
{\left[\mathbf{R}_{\mathbf{x}_{\mathbf{1}}, \mathbf{x}_{\mathbf{2}}}\right]_{m, n} } & =\sum_{i=1}^{D} \sum_{j=1}^{D} E\left(s_{i}\left(t+m \mathbf{u}\left(\theta_{i}\right)^{T} \mathbf{v}_{\mathbf{0}}\right) s_{j}^{*}\left(t-n \mathbf{u}\left(\theta_{j}\right)^{T} \mathbf{v}_{\mathbf{1}}\right)\right) \\
& +\sigma_{n}^{2} \delta(m-n) \\
& =\sum_{i=1}^{D} R_{s_{i}, s_{i}}\left(n \mathbf{u}\left(\theta_{i}\right)^{T} \mathbf{v}_{\mathbf{1}}+m \mathbf{u}\left(\theta_{i}\right)^{T} \mathbf{v}_{\mathbf{0}}\right)+\sigma_{n}^{2} \delta(m-n)
\end{aligned}
$$

where $R_{s_{i}, s_{i}}(\tau)$ denotes the autocorrelation of the $i$ th source signal at lag $\tau$ and (29) results because of the fact that the $i$ th and $j$ th signals are uncorrelated for $i \neq j$. We can rewrite (29) in terms of the power spectral density as

$$
\begin{aligned}
{\left[\mathbf{R}_{\mathbf{x}_{\mathbf{1}}, \mathbf{x}_{\mathbf{2}}}\right]_{m, n}=\frac{1}{2 \pi} } & \sum_{i=1}^{D} \int_{\omega_{a}}^{\omega_{b}} P_{s_{i}, s_{i}}(\omega) e^{j \omega\left(n \mathbf{u}\left(\theta_{i}\right)^{T} \mathbf{v}_{\mathbf{1}}+m \mathbf{u}\left(\theta_{i}\right)^{T} \mathbf{v}_{\mathbf{0}}\right)} d \omega \\
& +\sigma_{n}^{2}(m-n)
\end{aligned}
$$

where $P_{s_{i}, s_{i}}(\omega)$ is the power spectral density of the $i$ th wideband source. The next step is to vectorize $\mathbf{R}_{\mathbf{x}_{1}, \mathbf{x}_{2}}$. For this, define $\mathbf{S}(\omega)=\left[\begin{array}{ll}S_{1,1}(\omega) & S_{2,2}(\omega) \cdots S_{D, D}(\omega)\end{array}\right]^{T}$, and

$$
\mathbf{A}\left(\omega, \theta_{1}, \cdots, \theta_{D}\right)=\left[\mathbf{a}\left(\omega, \theta_{\mathbf{1}}\right) \cdots \mathbf{a}\left(\omega, \theta_{\mathbf{D}}\right)\right] \in \mathcal{C}^{M N \times D}
$$

where $\mathbf{a}\left(\omega, \theta_{\mathbf{i}}\right) \in \mathcal{C}^{M N \times 1}$ has the $(m+n N)$ th entry

$$
\left[\mathbf{a}\left(\omega, \theta_{i}\right)\right]_{m+n N, 1}=e^{j \omega\left(n \mathbf{u}\left(\theta_{i}\right)^{T} \mathbf{v}_{\mathbf{1}}+m \mathbf{u}\left(\theta_{i}\right)^{T} \mathbf{v}_{\mathbf{o}}\right)}
$$

Then we can rewrite (30) as

$$
\mathbf{y}=\frac{1}{2 \pi} \int_{\omega_{a}}^{\omega_{b}} \mathbf{A}\left(\omega, \theta_{1}, \cdots, \theta_{D}\right) \mathbf{S}(\omega) d \omega+\sigma_{n}^{2} \tilde{\mathbf{1}}_{n}
$$

Now, we wish to apply constant weight vector $\mathbf{w}$ to $\mathbf{y}$ in order to realize a given frequency invariant beampattern. Then the output of this beamformer is given by

$$
\begin{aligned}
z= & \sum_{i=1}^{D} \sum_{m=0}^{M-1} \sum_{n=0}^{N-1} \int_{\omega_{a}}^{\omega_{b}} w_{m, n}^{*} e^{j \omega\left(n \mathbf{u}\left(\theta_{i}\right)^{T} \mathbf{v}_{\mathbf{1}}+m \mathbf{u}\left(\theta_{i}\right)^{T} \mathbf{v}_{\mathbf{0}}\right)} P_{s_{i}, s_{i}}(\omega) \frac{d \omega}{2 \pi} \\
& +\sigma_{n}^{2} \mathbf{w}^{H} \tilde{\mathbf{1}}_{n}
\end{aligned}
$$

For frequency invariant beamforming, we need

$$
\sum_{m=0}^{M-1} \sum_{n=0}^{N-1} w_{m, n} e^{j \omega\left(n \mathbf{u}\left(\theta_{i}\right)^{T} \mathbf{v}_{\mathbf{1}}+m \mathbf{u}\left(\theta_{i}\right)^{T} \mathbf{v}_{\mathbf{0}}\right)}=G(\theta)
$$

where $G(\theta)$ is the desired frequency invariant beampattern. It can be readily observed that the above relation is equivalent to realizing (9) and hence the methods discussed in Section II can be used to realize this frequency invariant beampattern.

Summarizing, we have shown that using only $M+N$ physical sensors and performing beamforming with respect to the difference co-array, we can indeed avoid TDL filters and yet realize frequency invariant beams without increasing the number of sensors physically. 


\section{Examples}

We now provide design examples to validate the proposed virtual implementation of the lattice. We wish to implement a lattice with lattice generator vectors being both unit norm vectors with an angle of $45^{\circ}$ between them, and $M=N=17$. Hence we use two 17 element ULAs at an angle of $45^{\circ}$ with each other and perform the proposed covariance matrix based beamforming. To test the beampattern, we vary the direction of arrival of the impinging signal and plot the corresponding output of the beamformer. In order to test the frequency invariance of the beamformer, we transmit three narrowband signals, one at a time, with frequencies $\omega=\pi / 2,3 \pi / 4$ and $\pi$ and plot the resulting beampatterns in Fig. 1 using 200 snapshots to realize the covariance matrix. As can be seen, the beamformer output is fairly similar for all the three frequencies.



Fig. 1. Difference co-array based frequency invariant beampattern as a function of $\sin \theta$ using two 17 element ULAs plotted for the three frequencies $\omega=\pi / 2,3 \pi / 4$ and $\pi$, using 200 snapshots.

We provide another example as an application of this frequency invariant beamforming in suppressing wideband jammers. We assume the same array structure as in the previous example. However, to suppress jammers, we first realize a set of $P=7$ orthogonal frequency invariant beams spanning the beamspace, and then combine them by using suitable (narrowband) weights, to get the final output. The corresponding beamformer output as it evolves across various snapshots $(T)$, is plotted in Fig. 2. The jammer directions are assumed to be $\left\{60^{\circ}, 45^{\circ}, 30^{\circ}, 15^{\circ}\right\}$. It can be seen that for $T=300$ snapshots, clear nulls have been introduced in the beamformer output in the jammer directions.

\section{CONCluding Remarks}

In this paper, we proposed a novel way to realize frequency invariant beams (without TDL filters), which avoids the corresponding increase in the number of physical sensors by using the concept of co-arrays. This is made possible by a new kind of beamforming which filters the powers of the impinging signals instead of their amplitudes. This results in significant reduction in the number of sensors needed, yet achieving the desired frequency invariance. The simulation examples satisfactorily show the effectiveness of the proposed technique.
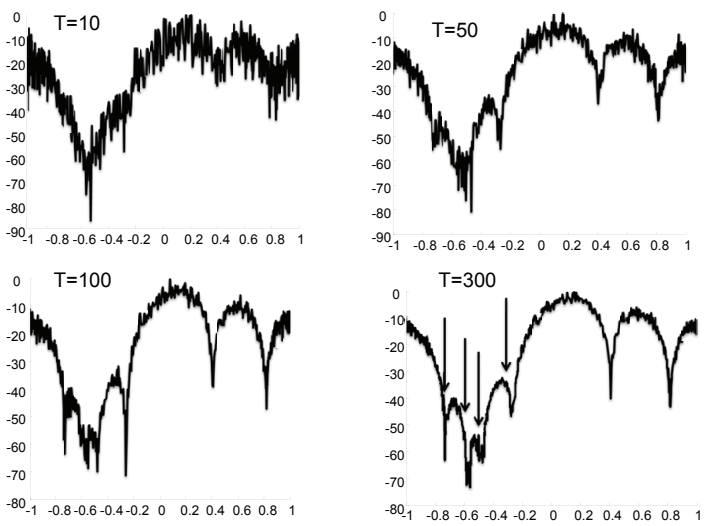

Fig. 2. Evolution of jammer nulling with snapshots $(T)$ based on the difference co-array using two 17 element ULAs at an angle $\psi=45^{\circ}$, with jammer directions given by $\left\{60^{\circ}, 45^{\circ}, 30^{\circ}, 15^{\circ}\right\}$.

\section{REFERENCES}

[1] S. C. Chan, and H. H. Chen, "Uniform Concentric Circular Arrays With Frequency-Invariant Characteristics-Theory, Design, Adaptive Beamforming and DOA Estimation," IEEE Trans. on Signal Processing, vol. 55, Issue 1, pp. 165-177, Jan. 2006.

[2] M. Ghavami, "Wideband smart antenna theory using rectangular array structures," Proc. IEEE Trans. on Signal Processing, vol. 50, Issue 9, pp. 2143-2151, Sept. 2002.

[3] R. T. Hoctor, S. A. Kassam, "The unifying role of the coarray in aperture synthesis for coherent and incoherent imaging," Proceedings of the IEEE, vol.78, no.4, pp.735-752, Apr 1990.

[4] Homer P. Bucker, "High resolution cross-sensor beamforming for a billboard array," J. Acoust. Soc. Am., Vol. 65, Issue 1, pp. 145-147, Jan. 1979.

[5] Homer P. Bucker, "Cross-sensor beam forming with a sparse line array," J. Acoust. Soc. Am., Vol. 61, Issue 2, pp. 494-498, Feb. 1977.

[6] W. Liu, D. McLernon, and M. Ghogho, "Frequency Invariant Beamforming Without Tapped Delay-Lines," IEEE International Conference on Acoustics, Speech and Signal Processing (ICASSP), 2007, vol. 2, pp. II-997 - II-1000, Apr. 2007.

[7] W. Liu, R. Wu, and R. J. Langley, "Design and Analysis of Broadband Beamspace Adaptive Arrays," IEEE Trans. on Antennas and Propagation, vol. 55, Issue 12, pp. 3413-3420, Dec. 2007

[8] Piya Pal, and P. P. Vaidyanathan, "Nested Arrays: A Novel approach to Array Processing with enhanced degrees of freedom," to appear in IEEE Transactions on Signal Processing.

[9] Hugh C. Pumphrey, "Design of Sparse Arrays in one, two and three dimensions," J. Acoust. Soc. Amer, vol. 93, Issue 3, pp. 1620-1628, March 1993.

[10] T. Sekiguchi, and Y. Karasawa, "Wideband beamspace adaptive array utilizing FIR fan filters for multibeam forming," IEEE Trans. on Signal Processing, vol. 48, Issue 1, pp. 277-284, Jan. 2000.

[11] M. Uthansakul, and M. E. Bialkowski, "Fully spatial wide-band beamforming using a rectangular array of planar monopoles," IEEE Trans. on Antennas and Propagation, vol. 54, Issue 2, Part 2, pp. 527-533, Feb. 2006.

[12] D. B. Ward, R. A. Kennedy, and R. C. Williamson, "Theory and design of broadband sensor arryas with frequency invariant far-field beam patterns, "Journal of Acoust. Soc. Amer., vol. 97, no. 2, pp. 1023-1034, Feb. 1995.

[13] D. B. Ward, R. A. Kennedy, and R. C. Williamson, "FIR filter design for frequency invariant beamformers, "IEEE Signal Processing Letters, vol. 3, pp. 69-71, Mar. 1996.

[14] D. B. Ward, Z. Ding, and R. A. Kennedy, "Broadband DOA Estimation using frequency invariant beamforming," IEEE Trans. on Signal Processing, vol. 46, no. 5, pp. 1463-1469, May 1998.

[15] H. L. Van Trees, Optimum Array Processing: Part IV of Detection Estimation and Modulation Theory. New York: Wiley Interscience, 2002. 